[19] M. O'Searcoid and T.T. West, Continuity of the generalized kernel and range of semi-Fredholm operators, Math. Proc. Cam. Phil. Soc. 105 (1989) 513-522.

[20] H. Radjavi and P. Rosenthal, Invariant subspaces (Ergebnisse der Math. 77, Springer 1973).

[21] A.Sinclair, Automatic continuity of linear operators (Cambridge University Press, UK 1976)

[22] T.T. West, A Riesz-Schauder theorem for semi-Fredholm operators, Proc. Royal Irish Acad. 87A (1987) 137-146.

University of Fairbanks,

Alaska, AK 99775

\section{Dimension Theory and Stable Rank}

\author{
Gerard J. Murphy
}

\section{Topological Dimension Theory}

The theory of dimension in topology grew from attempts to establish the the dimension of Euclidean spaces. The first proof that the spaces $\mathbb{R}^{n}$ and $\mathbb{R}^{m}$ are homeomorphic only if $n$ and $m$ are equal was iven by Brouwer in 1911. His proof did not explicitly involve a property that given same year Lebesgue might serve as a topological de to covering dimension. If $I$ is the closed suggested an approach which led to the covering dimension. If $I$ is the closed unit interval of $\mathbb{R}$ it was observed by Lebesgue that the cube $I^{n}$ can be covered by arbitrarily small closed sets in such a manner that not more than $n+1$ of them meet (in a common point). This is illustrated in the 2-dimensional case by the usual pattern of brickwork, where a maximum of 3 bricks can meet.

To define the covering dimension we introduce a preliminary concept. If $\Phi=\left(U_{\lambda}\right)_{\lambda \in \Lambda}$ is a family of subsets of a topological space. $X$ and $x \in X$ the arder of $\Phi$ at $x$, denoted ord $_{x}(\Phi)$, is defined to be the number of elements $\lambda$ o $\Lambda$ such that $U_{\lambda}$ contains $x$ (if there are infinitely many such elements $\lambda$ then $\left.\operatorname{ord}_{x}(\Phi)=+\infty\right)$. The order of $\Phi$ is defined to be the supremum of all ord $d_{x}(\Phi)$ where $x$ runs over $X$. Thus for the brickwork family of sets mentioned above where $x$ runs over $X$. Thus for the dimension of $X$ the order is 3 . If $X$ is a topological space the (covering) dimension of $X$ denoted $\operatorname{dim}(X)$, is the least integer $n$ such that every finite open covering $X$ has an open refinement of order not greater than $n+1$. If no such integer $n$ exists then we set $\operatorname{dim}(X)=+\infty$. Here is an alternative, very useful formulation: For any topological space $X$, the inequality $\operatorname{dim}(X) \leq n$ holds if and only if for each open covering $U_{1}, \ldots, U_{n+2}$ of $X$ there is an open covering $V_{1}, \ldots, V_{n+2}$ such that $V_{j} \subseteq U_{j}$ for $j=1, \ldots, n+2$ and $V_{1} \cap \ldots \cap V_{n+2}=\emptyset$ For the classical spaces such as $\mathbb{R}^{n}, I^{n}$ and $S^{n}$ (the $n$-sphere) the covering For the classical spaces such as $R^{n}, I^{n}$ and $S^{n}$ (the $n$-sphere) the covering dimension is the number one would expect. It is however theorem of Brouwer that $\operatorname{dim}\left(\mathbb{R}^{n}\right)=n$. The proof involves the well knot which asserts that for all $n$ the sphere $S$ 
ball of $\mathbb{R}^{n}$. There is a nice easily-stated criterion to determine if a subspace $X$ of a Euclidean space $\mathbb{R}^{n}$ has dimension $n$. This is true if and only if $X$ contains a non-empty open set of $\mathbb{R}^{n}$.

The case where the covering dimension is zero is easy to interpret in direct topological terms. If $X$ is Hausdorff and $\operatorname{dim}(X)=0$ then $X$ is necessarily normal and totally disconnected. For compact Hausdorff spaces being zerodimensional is equivalent to being totally disconnected, but this is not true for arbitrary spaces, as we shall see in an example below.

Incidentally, there are other concepts of dimension, such as the large and small inductive dimensions. For metric spaces the large inductive dimension is the same as the covering dimension, and all three dimensions agree on separable metric spaces. The covering dimension appears to be preferred for the analysis of general topological spaces.

There is a very nice connection between dimension theory and the problem of the existence of continuous extensions of certain continuous functions:

1.1. Theorem. If $X$ is a normal space then $\operatorname{dim}(X) \leq n$ if and only if for each closed set $A$ of $X$ and continuous function $f: A \rightarrow S^{n}$ there exists continuous extension $f^{\prime}: X \rightarrow S^{n}$.

Dimension theory also has useful applications to topological $\mathbb{K}$-theory. The latter topic can be viewed as the algebraic aspect of the theory of vector bundles, and one of the questions it investigates is the extent to which the cancellation property holds for (Whitney) direct sums of bundles. This is controlled in part by the dimension of the base space.

Although the covering dimension gives the expected answer in many classical situations, it has a number of paradoxical and even pathological features. For instance, the set $X=\{1,2,3,4\}$ can be endowed with a non-Hausdorff topology making it a topological space of covering dimension 1. This illustrates the point that dimension theory does not work too well for "strange" topological spaces. A mininum assumption appears to be normality to get some kind of reasonable theory. But even for compact Hausdorff spaces and metric spaces unexpected things can happen. For instance, the "logarithmic product rule"

$$
\operatorname{dim}(X \times Y)=\operatorname{dim}(X)+\operatorname{dim}(Y)
$$

fails spectacularly. To illustrate this denote by $Q$ the separable metric space of all square-summable sequences of rational numbers with the metric defined

by

$$
d\left(\left(x_{n}\right),\left(y_{n}\right)\right)=\left(\sum_{n=1}^{\infty}\left(x_{n}-y_{n}\right)^{2}\right)^{1 / 2} .
$$

One can show that $\operatorname{dim}(Q)=1$ and that $Q$ is homeomorphic to $Q \times Q$, so $(*)$ fails for $X=Y=Q$. (We promised above an example of a totally disconnected space not of dimension zero. The space $Q$ is such an example.) The logarithmic product rule fails even for compact spaces. Pontryagin has exhibited an example of a pair of compact metrisable spaces of dimension 2 whose product has dimension 3 . In a number of important cases one gets a useful inequality instead of $(*)$. If $X$ and $Y$ are arbitrary compact Hausdorff spaces then

$$
\operatorname{dim}(X \times Y) \leq \operatorname{dim}(X)+\operatorname{dim}(Y) .
$$

This inequality also holds in the case that $X$ and $Y$ are metric spaces.

Perhaps one should not really be surprised by these paradoxes of dimension theory. After all, as is well known, one can continuously map I onto $I^{2 !}$

There are many more aspects to dimension theory, a full account of which can be found in [5] and [6]. We turn now to the problem of defining the "dimension" of a $C^{*}$-algebra. We shall see there a number of possible candidates for the position, and all have connections with the classical topological dimension.

\section{The Bass Stable Rank and the Real Rank}

One of the great sucesses in recent years in operator algebra theory has been the development of K-theory of $\mathrm{C}^{*}$-algebras (for an introductory exposition see [3]). Recall that a $C^{*}$-algebra is a Banach algebra $A$ together with an isometric involution $a \mapsto a^{*}$ such that $\left\|a^{*} a\right\|=\|a\|^{2}$ for all $a \in A$. If $H$ is a Hilbert space and $B(H)$ denotes the algebra of all bounded linear operators on $H$ then $B(H)$ is a $\mathrm{C}^{*}$-algebra, as is every norm-closed self-adjoint subalgebra. A theorem of Gelfand and Naimark asserts that up to isomorphism these are all the $\mathrm{C}^{*}$-algebras.

There is an important connection with topology given as follows: If $X$ is a locally compact Hausdorff space then $C_{0}(X)$, the set of all continuous complexvalued functions on $X$ that vanish at infinity, is a commutative $\mathrm{C}^{*}$-algebra (the operations are pointwise-defined and the norm is the supremum norm). Conversely every commutative $\mathrm{C}^{*}$-algebra is of this form up to isomorphism. 
This correspondence between commutative $C^{*}$-algebras and locally compact Hausdorff spaces has inspired an approach to the theory of $\mathrm{C}^{*}$-algebras which regards it as "non-commutative topology", with the finitely-generated projective modules being the appropriate generalisation of vector bundles (based on a theorem of Swan). This point of view has been very fruitful and was the motivation for introducing $\mathrm{K}$-theory into $\mathrm{C}^{*}$-algebra theory. From this perspective it is natural to try to develop some concept of "dimension" for a $\mathrm{C}^{*}$-algebra analogous to the dimension of a topological space. This was attempted by Rieffel [10], who introduced the concept of the topological stable rank of a $C^{*}$-algebra. This was soon seen to be identical with the Bass stable rank already known to algebraists. Rieffel was led to investigate the stable rank by a question concerning a certain class of $\mathrm{C}^{*}$-algebras. If $\theta$ is an irrational number in $[0,1]$ then there is (up to isomorphism) a unique $\mathrm{C}^{*}$-algebra $A_{\theta}$ generated by a pair of unitaries $u, v$ such that $u v=e^{i 2 \pi \theta} v u$ ( $u$ is a unitary means that $u^{*}=u^{-1}$ ). These algebras, called irrational rotation algebras, are the motivating examples in the non-commutative differential geometry being developed by the Fields' medalist Alain Connes. The question that interested Rieffel is whether the cancellation property for projective modules holds for the irrational rotation algebras. This can be reformulated in more concrete terms: if two projections in $A_{\theta}$ have equal trace are they necessarily unitarily equivalent? The answer turns out to be affirmative, as was shown by Rieffel using the stable rank. He was led to his notion of topological stable rank by the following theorem of classical dimension theory:

2.1. Theorem. If $X$ is a compact Hausdorff space then its dimension is the least integer $n$ such every continuous function from $X$ to $\mathbb{R}^{n+1}$ can be uniformly approximated arbitrarily closely by continuous functions which never vanish.

Interpreting this in terms of the algebra $C_{0}(X)$, and then slightly reformulating, one arrives at the definition of stable rank. Let $A$ be a unital $\mathrm{C}^{*}$-algebra, and for each integer $n$ let $L_{n}(A)$ be the set of $n$-tuples $\left(a_{1}, \ldots, a_{n}\right)$ generating $A$ as a left ideal, that is, such that

$$
A a_{1}+\cdots+A a_{n}=A \text {. }
$$

The stable rank $\operatorname{sr}(A)$ of $A$ is the least integer $n$ such that $L_{n}(A)$ is dense in $A^{n}$ for the product topology. If no such integer exists $\operatorname{sr}(A)=+\infty$. If
$A=C_{0}(X)$ where $X$ is a compact Hausdorff space, and if $[t]$ denotes the integer part of $t$, then $\operatorname{sr}(A)=[\operatorname{dim}(X) / 2]+1$.

Except in the lowest rank case, it is not easy to interpret the stable rank directly in terms of properties of the algebra $A$. It can however be proved without difficulty that a unital $C^{*}$-algebra $A$ is of stable rank 1 if and only if without difficulty the ef invertible elements of $A$ is dense in $A$. The question of whether this the set of invertible for the irrational rotation algebras was open for a number of years, and has only recently been determined (in the affirmative) [9].

As in classical dimension theory, there are a number of surprising results in the theory of the stable rank. For instance, stable $\mathrm{C}^{*}$-algebras can have in the theory or 1 only. (A C $C^{*}$-algebra $A$ is stable if it is isomorphic to the $\mathrm{C}^{*}$ rank either 1 or 2 only. the Hilbert space $\ell^{2}$. These algebras occur frequently in $\mathrm{C}^{*}$-theory.) If $A$ is an the Hilber square matrices of size $n$ arbitrary $\mathrm{C}^{*}$-algebra and $M_{n}(A)$ denotes the set of square matrices of size having entries in $A$, then $M_{n}(A)$ is a $\mathrm{C}^{*}$-algebra in a natural way. If $\operatorname{sr}(A)=1$ then $\operatorname{sr}\left(M_{n}(A)\right)=1$, that is, if the invertible elements of $A$ are dense in $A$ then the invertible elements of $M_{n}(A)$ are dense in $M_{n}(A)$. If $\operatorname{sr}(A)=$

then $\operatorname{sr}\left(M_{n}(A)\right)=+\infty$ also. If $1<\operatorname{sr}(A) \leq n+1$ then $\operatorname{sr}\left(M_{n}(A)\right)=2$. Following along lines set down by Rieffel, Brown and Pedersen [1] intro-
duced another concept of rank for $\mathrm{C}^{*}$-algebras. If $A$ is a unital $\mathrm{C}^{*}$-algebra then its real rank is defined to be the smallest integer, $R R(A)$, such that for then its real rank $\left(a_{1}, \ldots, a_{n}\right)$ of self-adjoint elements of $A$ for which $n \leq R R(A)+1$ each $n$-tuple $\left(a_{1}, \ldots, a_{n}\right)$ of self-adjoint elements of $A$ for which $n \leq R R(A)+1$ such that $\sum_{k=1}^{n} b_{k}^{2}$ is invertible and

$$
\left\|\sum_{k=1}^{n}\left(a_{k}-b_{k}\right)^{2}\right\|<\varepsilon .
$$

(The element $a$ is self-adjoint if $a^{*}=a$.) If $X$ is a compact Hausdorff space and $A=C_{0}(X)$ then it is easy to show that $R R(A)=\operatorname{dim}(X)$. For an arbitrary $A=C_{0}(X)$ unital $C^{*}$-algebra $A$ the real and stable ranks are related by the inequality

$$
R R(A) \leq 2 \operatorname{sr}(A)-1
$$

However these two ranks can be very far apart. There is a $C^{*}$-algebra $A$ such the $R R(A)=0$ and $\operatorname{sr}(A)=+\infty$. Based on previous experience one would that $R R(A)=$ low expect the lowest real rank cas in $R R(A)=0$ if and only if every 
self-adjoint element of $A$ is the limit of a sequence of such elements having finite spectra. Algebras satisfying this condition had already been extensively analysed by Pedersen [8], and have some very interesting properties.

\section{The Analytic Rank}

Motivated by results in classical dimension theory the author has introduced a third concept of rank. If $A$ is a unital $C^{*}$-algebra a $C^{*}$-subalgebra $B$ is defined to be analytic if $B$ contains the unit 1 of $A$ and $a^{2} \in B$ implies $a \in B$ for all self-adjoint elements $a \in A$. If $S$ is an arbitrary subset of $A$ then there is a smallest analytic subalgebra of $A$ containing it, and if this is $A$ itself and the elements of $S$ are self-adjoint then $S$ is called an analytic base of $A$.

Every analytic subalgebra contains all the elements with finite spectrum in particular all the projections. If $A$ is of real rank zero then its only analytic subalgebra is $A$ itself. The field $\mathrm{C}$ is an analytic subalgebra of an arbitrary $\mathrm{C}^{*}$-algebra $A$ if and only if the only projections of $A$ are 0 and 1 . In particular if $A=C_{0}(X)$ then $\mathrm{C}$ is an analytic subalgebra if and only if $X$ is connected.

The analytic rank, $\operatorname{ar}(A)$, of a $C^{*}$-algebra $A$ is defined to be $+\infty$ if $A$ has no finite analytic base, and to be $n$ if $A$ has an analytic base of this (finite) cardinality but none of smaller cardinality. If $X$ is a compact metric space then by classical dimension theory results one has $\operatorname{ar}(A)=\operatorname{dim}(X)$. The analytic rank, considered purely as a $C^{*}$-algebra invariant, seems to behave better in a number of respects than the stable rank and the real rank, although like these it has some paradoxical properties.

Here is an example of nice behaviour. Associated with each locally compact group $G$ there is a $C^{*}$-algebra $C^{*}(G)$ having the same representation theory as $G$ (thus the representation theory of these groups is contained in the representation theory of $C^{*}$-algebras). If $\mathbf{F}_{n}$ is the (discrete) free group on $n>1$ generators then the stable rank of $C^{*}\left(\mathrm{~F}_{n}\right)$ is $+\infty$, so the stable rank unable to distinguish between these algebras. However $\operatorname{ar}\left(C^{*}\left(\mathbb{F}_{n}\right)\right)=n$

to have. For instance of properties that one would like a "rank" function to have. For instance, if $A \mapsto r(A)$ is a rank function it is desirable that it
should satisfy the following conditions:

(1) If $A=A_{1} \oplus A_{2}$ then $r(A)=\max \left\{r\left(A_{1}\right), r\left(A_{2}\right)\right\}$.

(2) If $B$ is a quotient algebra of $A$ then $r(B) \leq r(A)$.

(3) If $A=A_{1} \otimes_{*} A_{2}$, that is, $A$ is the (spatial) $\mathrm{C}^{*}$-tensor product of $A_{1}$ and $A_{2}$, then $r(A) \leq r\left(A_{1}\right)+r\left(A_{2}\right)$.

(4) If $A=B \times \times_{\alpha} G$, that is, $A$ is the $C^{*}$-crossed product of the unital
$C^{*}$-algebra $B$ and the countable discrete abelian group $G$, then $r(A) \leq$ $r(B)+\operatorname{dim}(\hat{G})$, where $\hat{G}$ is the Pontryagin dual group of $G$.

The analytic rank satisfies all four conditions, and the proofs involved in showing this are not difficult. However, some of these conditions are difficult, or unknown, for the stable and real ranks.

The analytic rank seems to be a natural invariant. However the concept of analytic $C^{*}$-subalgebra is rather mysterious, and it would be useful to be able to reformulate the definition of analytic rank in terms of better understood $\mathrm{C}^{*}$-algebraic ideas.

It appears that (at least some of) these various concepts of rank may be of great future importance. As yet however the theory is only in its initial stages. It is desirable to compute the ranks of many more examples, and to be able to interpret the ranks more directly in terms of properties of the algebras.

\section{References}

[1] L.G. Brown and G.K. Pedersen, C*-algebras of real rank zero, preprint (1989),

[2] R.H. Herman and L.N. Vaserstein, The stable range of $\mathrm{C}^{*}$-algebras, Inventiones Math. 77 (1984), 553-555.

[3] G.J. Murphy, Extensions and K-Theory of $\mathrm{C}^{*}$-algebras, Bull. Irish Math. Soc. 18 (1987), 18-29.

[4] G.J. Murphy, The analytic rank of a $C^{*}$-algebra, submitted.

[5] J. Nagata, Modern Dimension Theory. Heldermann Verlag, Berlin, 1983.

[6] A.R. Pears, Dimension Theory of General Spaces. Cambridge Univ. Press, Cambridge, 1975

[7] G.K. Pedersen, $C^{*}$-Algebras and their Automorphism Groups. Academic Press, New York-London, 1979.

[8] G.K. Pedersen, The linear span of projections in simple $\mathrm{C}^{*}$-algebras, J. Operator Theory 4 (1980), 289-296.

[9] I.F. Putnam, The invertible elements are dense in the irrational rotation $\mathrm{C}^{*}$-algebras, preprint (1989)

[10] M.A. Rieffel, Dimension and stable rank in the K-theory of $\mathrm{C}^{*}$-algebras, Proc. London Math. Soc. (3) 46 (1983), 301-333.

Department of Mathematics,

University College, Cork 